\title{
Le comportement d'interface sol-structure : aspects expérimentaux et numériques
}

\section{Basic features of soil-structure inferface behaviour}

\author{
M. BOULON \\ Maître de Conférences à l'Université Joseph-Fourier de Grenoble \\ Chercheur à l'institut de Mécanique de Grenoble, UMR 101*
}

Rev. Franç. Géotech. n 54, pp. 27-37 (janvier 1991)

\section{Résumé}

L'auteur présente tout d'abord quelques données concernant le frottement latéral le long des pieux ancrés dans le sable pour ensuite examiner l'essai élémentaire correspondant (un test de cisaillement direct entre sol granulaire et matériau de construction). L'influence des conditions initiales et le chemin imposé sont mis en évidence.

Le comportement correspondant est décrit grâce à un modèle d'interface utilisant les concepts de dépendance directionnelle et d'interpolation rhéologique ; dans cette approche, les chemins de base sont des essais de cisailiement direct à contrainte normale constante, à volume constant, ainsi que des chemins pseudo-œdométriques. La loi incrémentale correspondante, entre contraintes de contact et déplacements relatifs, est intégrée numériquement le long de divers chemins et comparée à des essais de cisaillement direct à rigidité normale imposée. On montre que la contrainte de cisaillement maxima mobilisée est une fonction monotone de ce paramètre de rigidité.

Une application par la méthode des éléments finis, comparant les prédictions de divers modèles d'interface, dont le précédent, montre l'influence du paramètre principal qu'est le taux de dilatance.

Une évaluation simplifiée du frottement latéral mobilisé le long des pieux, ancrages, clous, est ensuite proposée en vue de l'utilisation dans la méthode des courbes $(t-z)$. Une attention particulière est portée à la détermination de ces courbes, soit à partir d'essais spéciaux de cisaillement direct, soit à partir du modèle théorique d'interface.

\section{Abstract}

Firstly experimental data of friction along piles in sand are presented; the corresponding element test (direct shear test between a granular soil and a rough construction material) is described, showing the effect of the initial conditions and of the prescribed path.

The phenomenon is described by a mathematical model of interface behaviour which employs the concepts of directional dependence and rheological interpolation. The element tests used in that approach are direct shear tests at constant normal stress, at constant volume, and (pseudo) oedometers. The resulting incremental relationship between contact stresses and relative dispacements is numerically integrated for several loading conditions and compared with direct shear experiments at prescribed normal stiffness. It is shown that the maximum mobilized shear stress during such a test can be considered as a monotonic function of this stiffness parameter.

A finite element application comparing the predictions of various interface constitutive equations (among them the previous one) shows the influence of the main parameter being the dilatancy rate.

A simplified evaluation of the mobilized friction shear along piles, anchors, nails, is then proposed for use in the so-called $\mathrm{t}-\mathrm{z}$ curves method. Attention is focussed on the determination of these curves, either from special direct shear tests or from the theoretical interface model. 


\section{INTRODUCTION}

L'examen d'essais d'arrachement de pieux ou d'ancrages in situ, ou d'essais sur pieux-modèles en laboratoire pose question quant à la modélisation des phénomènes observés. Par exemple, le cisaillement mobilisé durant un essai d'arrachement peut présenter un écart considérable par rapport aux prédictions supposant un état initial " au repos ", Ko, autour de l'inclusion. Dans le cas des pieux battus dans le sable par exemple, COYLE et CASTELLO [6] ont mesuré des valeurs élevées du coefficient $\mathrm{K}$ de pression latérale des terres sur le fût (plus de 1,8), Bien entendu, cet effet mesuré par de nombreux auteurs doit être mis en rapport avec une contrainte normale latérale, induite pendant l'arrachement, bien supérieure à celle de l'état Ko. Une interprétation équivalente est basée sur le concept de coefficient de frottement apparent $\operatorname{tg} \delta^{*}$, développé par SCHLOSSER et ELIAS [16]. GUILLOUX et al. [8], et qui décroît avec le niveau de contrainte.

Des essais très fiables d'ancrages, réalisés en laboratoire par WERNICK [17], et de pieux modèles par PUECH et al. [12], montrent que cet effet existe même pour les inclusions moulées. L'ensemble de ces observations prouve que le système de contraintes latérales varie le long des inclusions non seulement pendant leur phase d'installation (ce qui se conçoit bien dans le cas des pieux battus et foncés), mais aussi sous charge de service. Nous nous proposons de développer ci-dessous un modèle d'interface capable de décrire le frottement latéral le long des inclusions ancrées dans les sols grossiers, dans le cas « moulé », processus d'installation excluant les grandes déformations. Ce modèle fait suite à nos études précédentes, et s'en distingue par le fait que nous sommes maintenant en mesure de décrire les fortes contractances (chutes de résistance) dues à la rupture des grains.

\section{L'ESSAI ÉLÉMENTAIRE}

Depuis fort longtemps, FEDA [7] a décrit l'essai de cisaillement direct comme une simulation physique élémentaire du frottement latéral le long des inclusions dans le sol. SCHLOSSER et GUILLOUX [15] ont étudié les relations entre le cisaillement direct à volume constant et le frottement latéral le long des ancrages. POTYONDY [13] et plus tard BRUMMUNDS et LEONARDS [4] ont examiné les propriétés de contact entre le sable et divers matériaux de construction rugueux, à partir d'essais de cisaillement direct à contrainte normale constante dans un appareil de cisaillement modifié (l'une des demi-boîtes étant remplacée par le matériau de construction). Le premier problème à se poser est celui de l'homogénéité du test : on admet généralement que l'essai de cisaillement direct n'est pas fiable parce que contraintes et déformations ne sont pas homogènes au sein de l'échantillon; cette affirmation concerne l'échantillon en tant que milieu continu équivalent; en effet, si le test est considéré comme un essai relatif à une sur- face de contact (en raison de l'intense localisation) et non comme un essai relatif au volume entier de l'échantillon, le critère d'homogénéité est requis seulement le long de la surface de discontinuité de déplacement. De plus, l'expérience montre que la bande de cisaillement naissant au cours du cisaillement direct reste concentrée dans son domaine d'origine. En conséquence, nous adapterons le critère d'homogénéité en le souhaitant vérifié dans la bande de cisaillement seulement. De nombreux essais visualisés (rayons $X$ par SCARPELLI et WOOD [14], stéréophotogramétrie par $\mathrm{ClCHY}$ et al. [5]) ont montré que cette condition est bien réalisée dans l'essai de cisaillement direct, sauf au tout début de l'essai. Mais ce résultat cinématique n'exclut pas une non homogénéité possible concernant le niveau de contrainte au sein de la bande de cisaillement générée dans l'appareil de cisaillement direct plan. Notons d'ailleurs que la \& vraie boîte de cisaillement " proposée par WERNICK [17] ne peut qu'accroître cette hétérogénéité.

Le comportement d'interface sol-structure considéré comme un comportement de surface ou de contact avec frottement (il s'agit alors d'un milieu continu bidimensionnel) est une approche pragmatique adoptée afin de décrire la cinématique fort complexe (grandes déformations, grandes rotations, grands déplacements relatifs et grandes rotations des grains euxmêmes) quí intervient entre les deux faces de la bande de cisaillement située dans le sol, le long de la structure, dans le cas bidimensionnel représenté figure 1 .

La bande de cisaillement constituant l'interface solstructure ne peut être isolée sous peine de modification irrémédiable de son comportement. C'est la raison pour laquelle l'essai de cisaillement direct doit être interprété en considérant deux parties de l'échantillon (cf. fig. 2) : la partie inférieure * active * qui est en contact avec le matériau de construction rugueux et qui constitue l'interface, et la partie supérieure, « passive $\triangleright$, choisie aussi fine que possible mais non inexistante, et qui est sollicitée comme un échantillon oedométrique. On notera que actif et passif n'ont rien à voir avec les termes utilisés par les praticiens de la pression des terres.

Etant donné le choix réalisé d'un milieu bidimensionnel équivalent, les variables d'interface ne sont pas

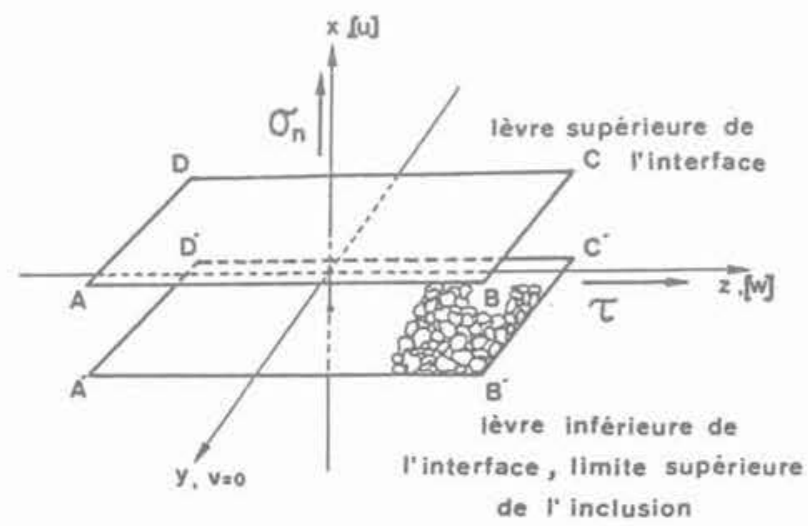

Fig. 1. - Idéalisation de l'interface sol-structure. Fig. 1. Idealized soil-structure interface, - 


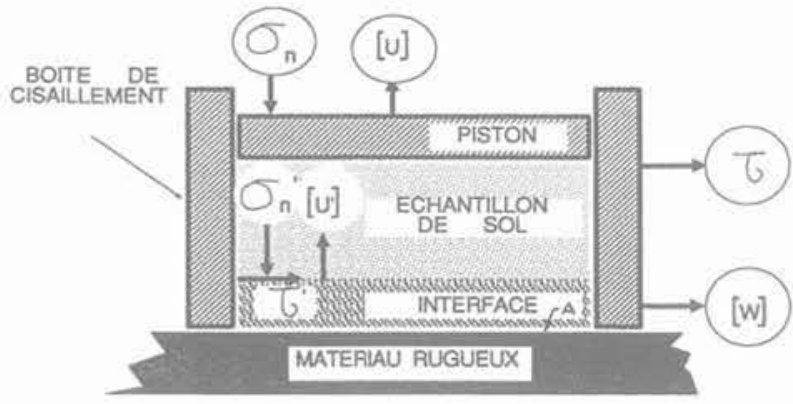

Fig. 2. - Interprétation de l'essai de cisaillement direct.

Fig. 2. - An interpretation of the direct shear test.

tensorielles mais vectorielles : ce sont d'une part le vecteur contrainte (l'opérateur n'a accès qu'à une moyenne sur la surface de contact (A) :

$$
\underline{\sigma}=\frac{1}{\mathrm{~A}}\left\{\begin{array}{l}
\int_{\mathrm{A}} r^{\prime} \mathrm{dA} \\
\int_{\mathrm{A}} \sigma_{\mathrm{n}}^{\prime} \mathrm{dA}
\end{array}\right\}\left\{\begin{array}{c}
\tau \\
\sigma_{n}
\end{array}\right\}
$$

et d'autre part le vecteur déplacement relatif entre les deux lèvres de l'interface :
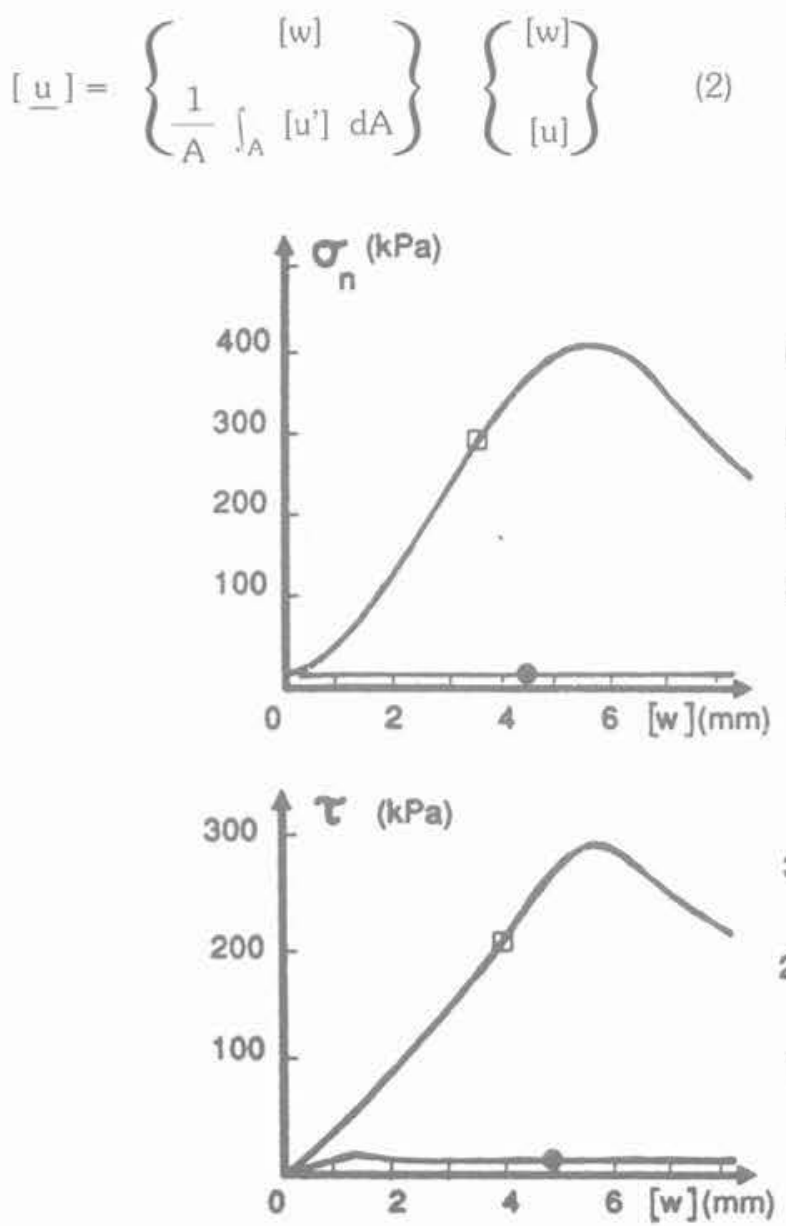

- Test (S)
La boîte de cisaillement annulaire (YOSHIMI et KISHIDA [18]) est certainement un outil plus satisfaisant que la boîte plane pour identifier le comportement d'interface, du point de vue des effets de bords, malgré une hétérogénéité radiale inhérente à l'appareil. L'essai de cisaillement direct à contrainte normale constante est si populaire que l'on oublie souvent que des conditions différentes peuvent être imposées à cet essai ; d'autres chemins peuvent être réalisés grâce à la boîte de cisaillement : ainsî, les tests à contrainte normale constante (S) et à volume constant (V) peuvent être considérés comme des chemins de cisaillement extrêmes. Sur la figure 3 sont représentés des résultats concernant le sable grossier siliceux d'Hostun $\left(\mathrm{d}_{50}=0,74 \mathrm{~mm}\right)$, à densité initiale élevée $(\gamma=$ $16,7 \mathrm{kN} / \mathrm{m}^{3}, \mathrm{D}_{\mathrm{r}}=0,9$ ) et faible contrainte normale initiale $\left(\sigma_{Y_{5}}=12,5 \mathrm{kPa}\right)$. Le matériau de construction rugueux a été obtenu par collage de sable identique sur une plaque métallique. La grande différence existant entre les deux tests (d'où lappelation d'essais extrêmes) est évidente, spécialement du point de vue de la contrainte de cisaillement mobilisée à déplacement relatif tangentiel égal, ou encore si l'on compare l'évolution du vecteur contrainte dans le plan $\left(\tau, \sigma_{n}\right)$; lors de l'essai $(\mathrm{V})$, l'extrémité du vecteur contrainte remonte la courbe intrinsèque résiduelle après l'avoir traversée en raison de la forte densité initiale du sable, puis redescend le long de celle-ci ; si l'essai se poursuivait (l'amplitude du déplacement relatif tangentiel est limitée à $30 \mathrm{~mm}$ sur la boîte de cisaillement plan) la liquéfaction statique pourrait être atteinte. Ces tests mettent en évidence les aspects
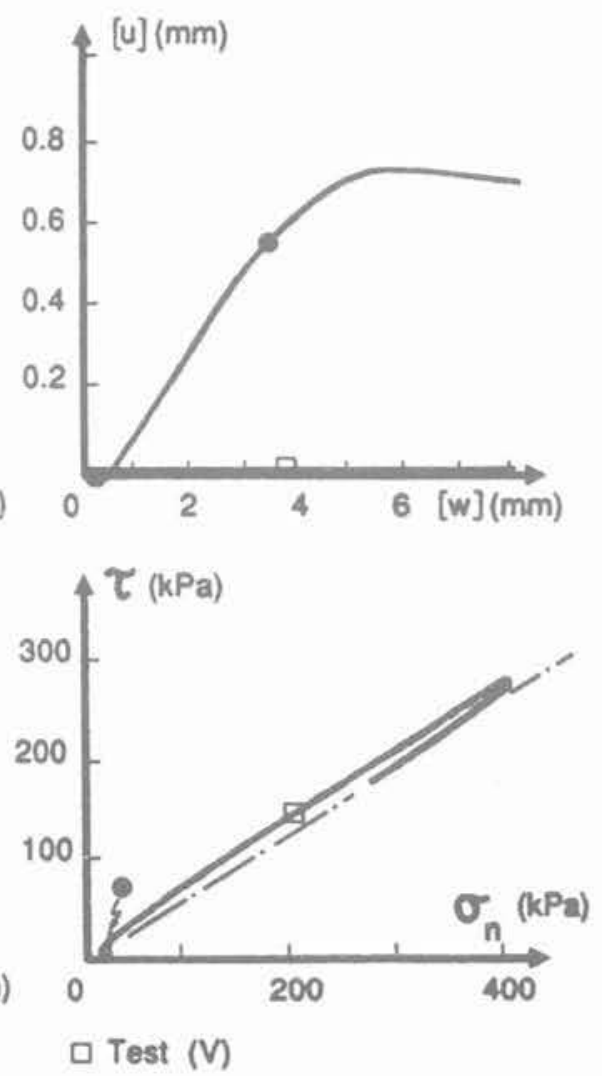

Fig. 3. - Essais de cisaillement extêmes sur sable d'Hostun dense à faible contrainte normale initiale.

Fig. 3. - Extremal direct shear tests on dense Hostun sand, at low initial normal stress. 
cinématiques et statiques de la dilatance localisée, mais également le comportement contractant du même sable lors d'un cisaillement de grande amplitude, c'est-à-dire après le pic du test (V). La figure 4 permet d'observer le comportement du même sable selon les chemins (S) et (V), à partir de conditions initiales "opposées $*$, c'est-à-dire faible densité initiale $\left(\gamma=15,4 \mathrm{kN} / \mathrm{m}^{3}, \mathrm{D}_{\tau}=0,3\right)$ et contrainte normale initiale élevée $\left(\sigma_{T_{n}}=1061 \mathrm{kPa}\right)$. La tendance contractante est ici prépondérante, rendant les tests (S) et (V) sensiblement équivalents en ce qui concerne le cisaillement maximum mobilisé. L'ensemble des figures 3 et 4 permet de souligner la différence essentielle existant entre l'essai triaxial non drainé et l'essai de cisaillement direct à volume constant: l'intense rupture de grains liée à la localisation de l'énergie plastique dans la bande de cisaillement (lors du cisaillement direct) induit un effet contractant permanent (dès le début de l'essai, même si les modifications de l'assemblage des grains masquent le phénomène. HOTEIT [9] - Ceci exclut évidemment toute notion d'état critique. On remarquera toutefois que globalement, toute perte de matériau pendant l'essai peut apparaître comme une contractance ; sans modification d'assemblage : ce défaut est possible, quoique limité, avec la boite de cisaillement plan.

\section{MODÈLE D'INTERFACE SOL-STRUCTURE}

Le paragraphe précédent nous a permis de mettre en évidence les aspects du comportement liés au che- min pour des matériaux non visqueux comme les sables. Nous avons développé les bases d'un modèle tridimensionnel d'interface sol-structure incluant les axes matériels et l'outil expérimental susceptible de donner accès aux paramètres du modèle (BOULON [13]) : nous nous attacherons ici à décrire le cas bidimensionnel, plus couramment utile. Nous considérons les vitesses de contrainte et de déplacement relatif tangentes au chemin courant

$$
\underline{\dot{\sigma}}=\left\{\begin{array}{c}
\dot{\tau} \\
\dot{\sigma_{n}}
\end{array}\right\} \quad \underline{\underline{u}]}=\left\{\begin{array}{c}
{[\dot{w}]} \\
{[\dot{u}]}
\end{array}\right\}
$$

Une définition unique du chemin et la nécessité de comparaison des chemins entre eux suggèrent une normalisation du chemin tangent. Un choix formel doit également être réalisé en vue de définir la sollicitation tangente et la réponse tangente. Les comportements donnant naissance à des pics de contraintes interdisent de choisir la vitesse de contrainte comme sollicitation tangente. Les chemins tangents sont donc normés par:

$$
\| \underline{\underline{u}}] \|=\left([\dot{w}]^{2}+[\dot{u}]^{2}\right)^{1 / 2}
$$

d'où les paramètres réduits caractérisant le chemin tangent :

sollicitation : $\left\{\begin{array}{c}\lambda \\ \mu\end{array}\right\}=\frac{1}{\|[\underline{\mathrm{u}}]\|}\left\{\begin{array}{l}{[\dot{\mathrm{w}}]} \\ {[\dot{\mathrm{u}}]}\end{array}\right\}$
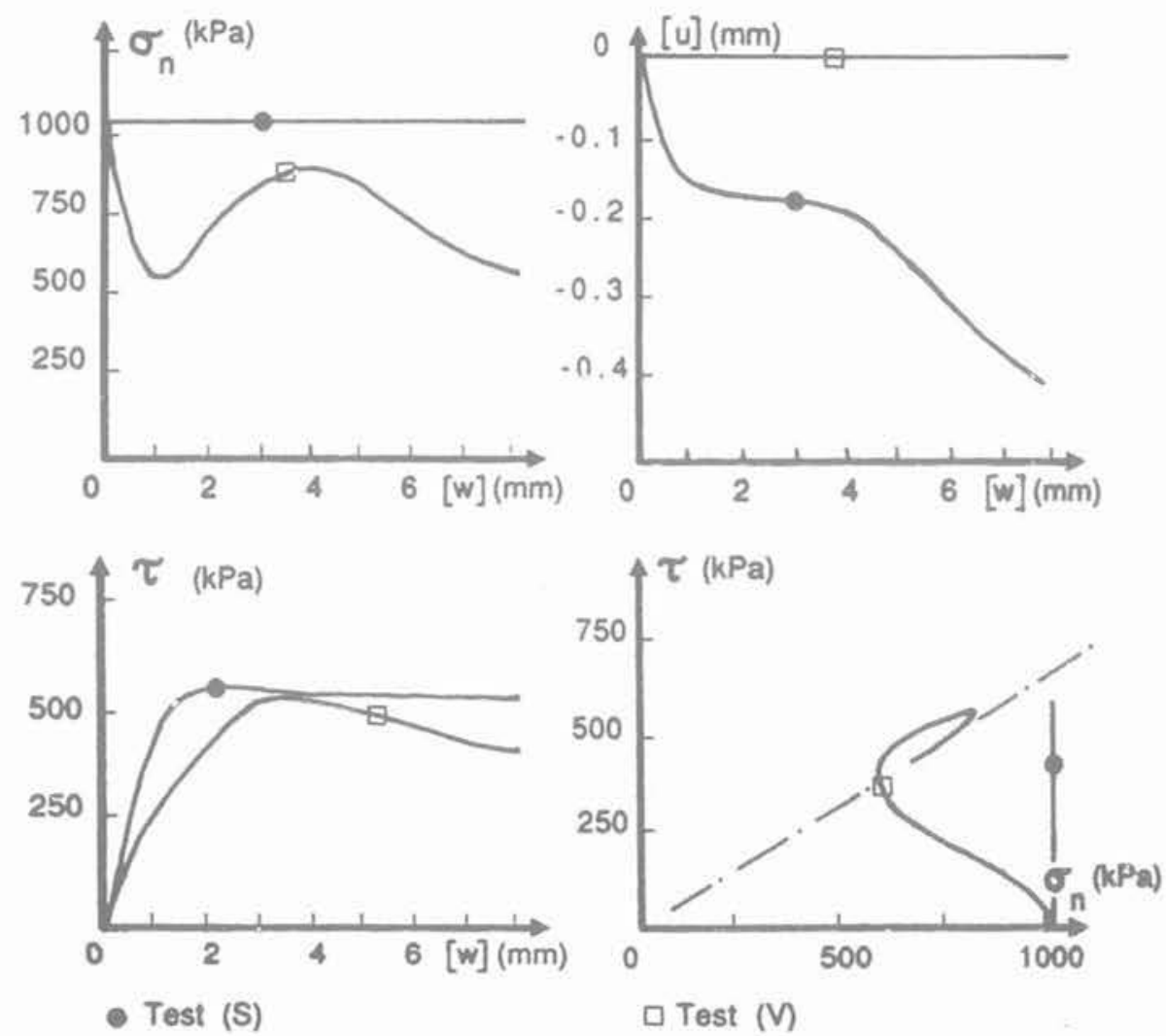

Fig. 4. - Essais de cisaillement extrêmes sur sable d'Hostun lâche à contrainte normale initiale élevée. Fig. 4. - Extremai direct shear tests on loose Hostun sand, at high initial normal stress. 
réponse: $\left\{\begin{array}{l}\xi \\ \eta\end{array}\right\}=\frac{1}{\|[\underline{u}]\|}\left\{\begin{array}{l}\tau \\ \sigma_{n}\end{array}\right\}$

Dans l'espace des sollicitations incrémentales $(\lambda, \mu)$. tous les chemins possibles sont les points du cercle unité. Les points correspondants de l'espace des réponses incrémentales $(\xi, \eta)$ sont paramétrés par $\lambda$ et $\mu$ liés par une relation $\left(\lambda^{2}+\mu^{2}=1\right.$, équivalente à (4)) ; ils appartiennent donc à la courbe de réponse (e) présentée à la figure 5 .

Afin de préciser cette réponse incrémentale, il est capital d'utiliser l'ensemble des informations possibles; en d'autres termes, il convient de connaitre des points représentatifs de (e) afin de générer la partie inconnue de celle-ci. Dans le cas bidimensionnel, la boîte de cisaillement direct permet des mesures aisées selon six chemins (trois en charge et trois en décharge) (cf. fig. 5). Les chemins 1 à 4 ont été présentés précédemment; les chemins 5 et 6 sont appelés pseudoœdométriques, c'est-à-dire qu'ils représentent des œdomètres après cisaillement, soit la limite d'essais de cisaillement $(\lambda=0)$.

La direction de sollicitation tangente est ainsi définie dans lespace $(\lambda, \mu)$. La partie inconnue de (e) est générée par interpolation directionnelle, l'argument de cette interpolation étant la distance angulaire de l'espace $(\lambda, \mu)$.

Supposons que nous cherchions la réponse incrémentale $(\xi, \eta)$ correspondant à un chargement incrémental $(\lambda, \mu)$ donné ; ce chargement est représenté par le point $\mathrm{D}$, d'angle polaire $\beta$ dans l'espace $(\lambda, \mu)$ (cf. fig. 6). Chaque chemin de base (repéré par

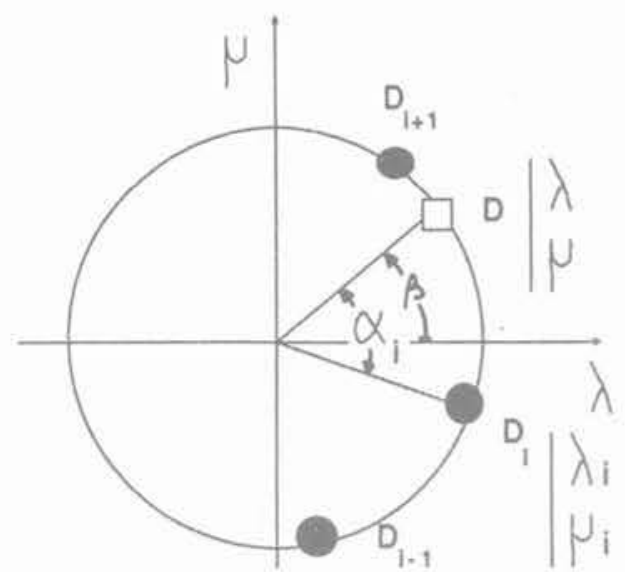

Fig. 6. - Les paramètres de l'interpolation directionnelle.

Fig. 6. - The parameters of the directional imterpolation.

l'indice i) est représenté par le point Di correspondant. Soit $\alpha_{i}$ l'angle arithmétique séparant les directions $(\lambda$, $\mu$ ) et $\left(\lambda_{i}, \mu_{i}\right)$; la réponse tangente est définie comme la somme pondérée:

$$
\left\{\begin{array}{l}
\xi \\
\eta
\end{array}\right\}=\sum_{i=1}^{N} W_{i}\left\{\begin{array}{l}
\xi_{i} \\
\eta_{i}
\end{array}\right\}
$$

avec :

$$
\left\{\begin{array}{l}
\xi_{i} \\
\eta_{i}
\end{array}\right\} \neq\left\{\begin{array}{c}
\xi_{j} \\
\eta_{j}
\end{array}\right\}_{v i \neq j}
$$
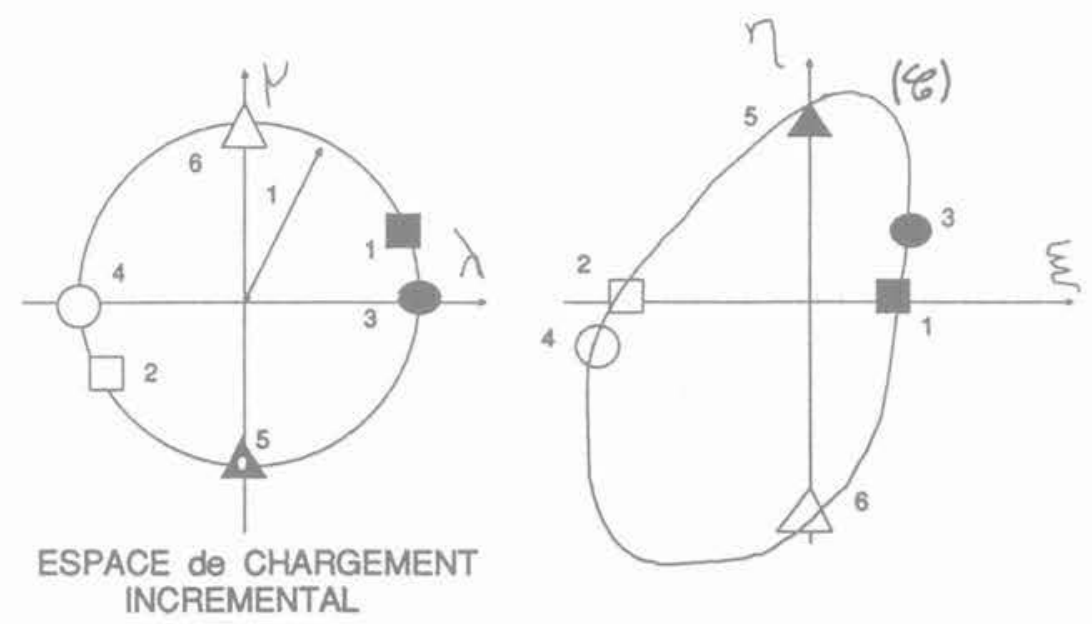

\section{ESPACE de REPONSE INCREMENTALE}

1 Contrainte normale constante, en charge

2 Contrainte normale constante, en décharge

3 Volume constant, en charge

4 Volume constant, en décharge

5 Pseudo-œdomètre, en charge

6 Pseudo-œdomètre, en décharge

Fig. 5. - Chargement incrémental et réponse incrémentale. Fig. 5 . - Incremental loading and incremental response. 
Les $\mathrm{N}$ fonctions de pondération Wi sont solution du système algébrique linéaire d'équations caractérisant une interpolation :

$$
\left\{\begin{array}{l}
\sum_{i=1}^{N} W_{i}=1 \\
W_{1} \alpha_{1}{ }^{x} y_{1}=W_{2} \alpha_{2}{ }^{x} y_{2}=\ldots=W_{N} \alpha_{N}{ }^{x} y_{N}
\end{array}\right.
$$

yi est une fonction de $\beta$ choisie de manière à assurer des valeurs positives et négatives pour $\mathrm{Wi}$. Le choix le plus simple est la fonction signe:

$$
\left\{\begin{array}{l}
\beta_{i-1} \leqslant \beta \leqslant \beta_{i+1}: y_{i}=+1 \\
\beta<\beta_{i-1}, \beta>\beta_{i+1}: y_{i}=-1
\end{array}\right.
$$

D'autres tentatives utilisant des fonctions splines à plusieurs déterminations ont donné de bons résultats dans le cas où les directions des chemins de base sont mal réparties notamment (MARCHINA [10]). La définition précédente est intéressante du point de vue de la continuité de la réponse (la continuité $\mathrm{C} 2$ est requise pour les fonctions $\mathrm{Wi}$ ).

La présentation générale de l'interpolation fait appel à six chemins de base. Dans certaines situations, on utilise moins de six chemins : le premier cas est relatif à celui des chemins de base très proches; par exemple, un chemin incrémental (S) correspondant à une dilatance très faible est évidemment très proche d'un chemin (V) ; une procédure doit être utilisée, prenant en compte le chemin * moyen * entre ceux$\mathrm{ci}$, afin d'éviter des difficultés numériques.

Le second cas concerne un chargement incrémental à partir d'un état de contrainte situé sur la courbe intrinsèque : aucun chemin de base ne peut correspondre à une sortie du domaine possible pour les vecteurs contrainte; d'où ici encore une technique spéciale pour éliminer les chemins physiquement inexistants.

S'il s'avère que d'autres chemins, aujourd'hui non réalisables en laboratoire, mais disponibles demain..., soient très caractéristiques du cisaillement direct, ils pourront évidemment être incorporés dans cette formulation, puisque le nombre $\mathrm{N}$ de chemins de base n'est évidemment pas limité à 6 .

Il est bien connu en rhéologie que la question des variables gouvernant l'état du matériau est l'une des plus délicates à résoudre, et ceci sans données mathématiques, excepté quelques lointaines limites thermodynamiques. Dans notre cas, les chemins de base sont formulés analytiquement, en vue d'une dérivation pour obtenir les chemins tangents (incrémentaux), en utilisant comme ensemble de paramètres d'état $s_{k}$ les variables actuelles d'interface $\left([w],[u], \tau, \sigma_{n}\right)$ et une densité supposée $\gamma$ (sous contrainte) au sein de l'interface (MARCHINA [10]). Au vu des succès obtenus en mécanique des joints rocheux, par PLESHA [11] notamment, nous avons l'intention de décrire les modifications de densité sous contrainte nulle $\left(\gamma_{0}\right)$ grâce à l'énergie spécifique Ws (t) - t étant le temps, dont l'origine est située au début de l'essai.

$$
\mathrm{W}_{\mathrm{s}}(\mathrm{t})=\int_{\theta=0}^{\theta=\mathrm{t}}\left\{\tau \mathrm{d}[\mathrm{w}]-\sigma_{\mathrm{n}} \mathrm{d}[\mathrm{u}]\right\}
$$

Cette densité est évidemment à relier à la rupture des grains évoquée précédemment.

L'état actuel de l'interface étant connu par $s_{\mathrm{k}}$, et la direction de la sollicitation incrémentale par [w] et [u] (ou $\lambda$ et $\mu$ ), l'interpolation rhéologique sur les chemins tangents de base conduit à :

$$
\left\{\begin{array}{c}
\tau \\
\sigma_{n}
\end{array}\right\}=\left\{\begin{array}{l}
\tau\left([\dot{w}],[\dot{u}], s_{k}\right) \\
\dot{\sigma}_{n}\left([\dot{w}],[\dot{u}], s_{k}\right)
\end{array}\right\}
$$

Supposant le matériau totalement non visqueux, c'està-dire non sujet à une réponse différée, et à réponse directionnellement dépendante $\left(\tau\right.$ et $\dot{\sigma}_{\mathrm{n}}$ sont alors des fonctions homogènes de degré 1 en [ï] et [u]), les composantes de l'équation constitutive tangente sont données par le théorème d'Euler sur les fonctions homogènes:

$\left\{\begin{array}{c}\dot{\tau} \\ \dot{\sigma}_{\mathrm{n}}\end{array}\right\}=\left[\begin{array}{ll}\frac{\partial \dot{\tau}}{\partial[\dot{w}]} & \frac{\partial \dot{\tau}}{\partial[\dot{u}]} \\ \frac{\partial \dot{\sigma_{n}}}{\partial[\dot{w}]} & \frac{\partial \dot{\sigma}_{n}}{\partial[\dot{u}]}\end{array}\right] \times\left\{\begin{array}{c}{[\dot{w}]} \\ {[\dot{u}]}\end{array}\right\}$

Ces quatre composantes fonctions de la direction $(\lambda$, $\mu$ ) ont une expression analytique résultant de la dérivation des fonctions de pondération $\mathrm{Wi}$ et des pentes locales des chemins de base.

\section{INTÉGRATION NUMÉRIQUE DE LA LOI DE COMPORTEMENT}

Lorsque le chemin est donné explicitement par $[\dot{w}(t)]$ et $[\dot{u}(t)]-$ ou $\lambda(t)$ et $\mu(t)-, t$ étant le temps, la réponse tangente est directement calculée ; ce n'est généralement pas le cas, et une recherche itérative de la direction locale de fonctionnement, correspondant aux données réelles de la sollicitation est alors nécessaire; ceci met en lumière le caractère formel et relatif des notions précédemment adoptées de sollicitation et de réponse.

La loi de comportement (11) a été principalement intégrée par méthode explicite à pas constant le long de chemins de cisaillement à rigidité normale imposée ; ces chemins sont extrêmement significatif́s car ils caractérisent le frottement latéral le long des pieux et autres inclusions ancrées dans le sol. De plus, les essais correspondants de cisaillement direct peuvent être réalisés en laboratoire. Notre modèle a été testé pour le sable d'Hostun moyen $\left(\mathrm{d}_{50}=0,74 \mathrm{~mm}\right)$ à partir des conditions initiales suivantes :

$$
\begin{aligned}
& \gamma_{\circ}=17,1 \mathrm{kN} / \mathrm{m}^{3} \text { (dense) }: \sigma_{\mathrm{n}_{0}}=124 \mathrm{kPa} \\
& \text { DR }=0-9
\end{aligned}
$$

Des résultats expérimentaux et numériques typiques (BOULON et al. [1]) de $\tau$ et $\sigma_{\mathrm{n}}$ fonction de [w] sont présentés ci-après (fig. 7), pour deux rigidités normales imposées $(\mathrm{k}=5000 \mathrm{kPa} / \mathrm{mm}$ et $40000 \mathrm{kPa} / \mathrm{mm}$ ). 

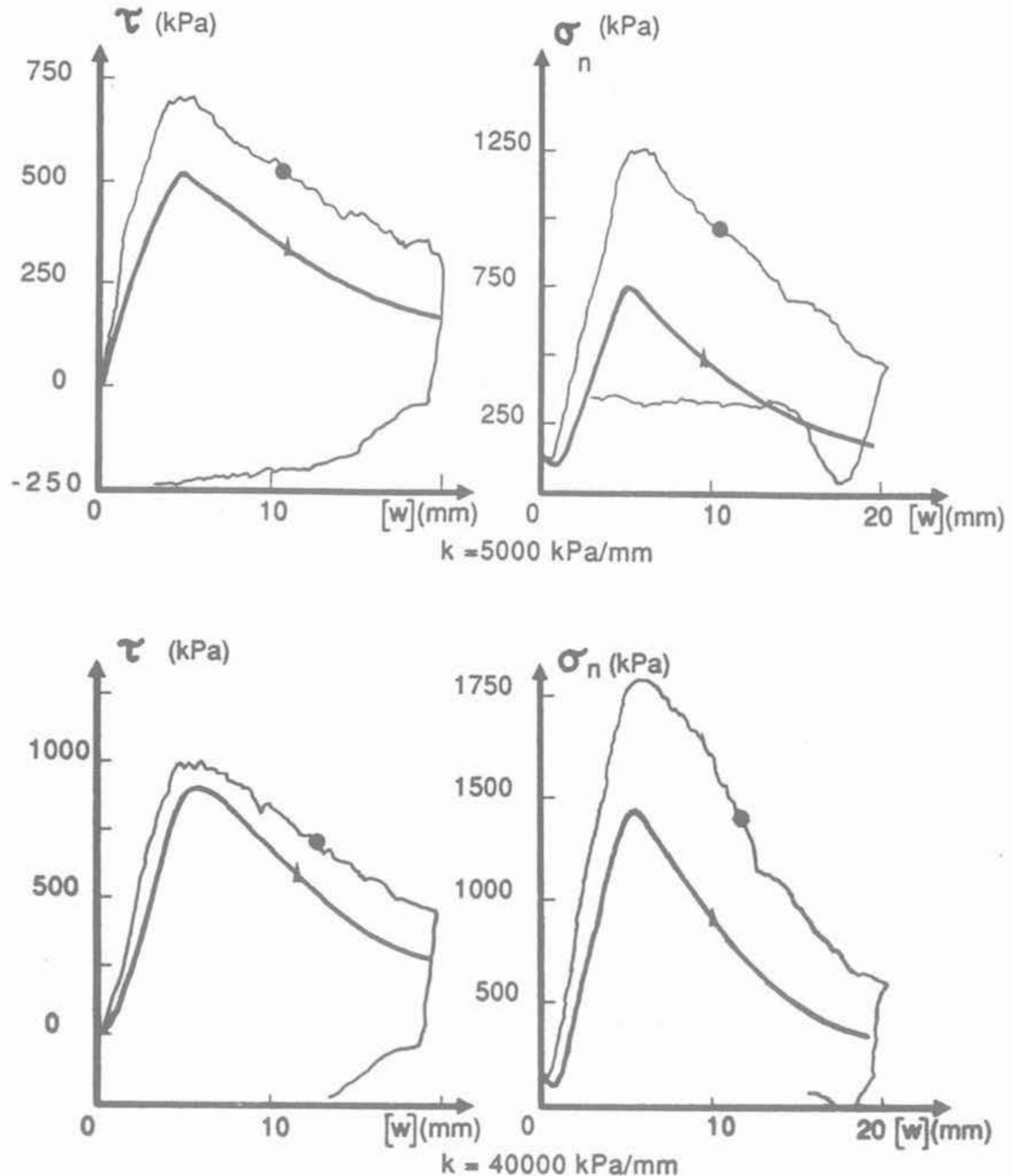

Fig. 7. - Chemins typiques de cisaillement direct à rigidité normale imposée $(k)$; expérience $\bullet$ et simulation $\mathbf{A}$; sable d'Hostun dense, $\sigma_{n_{0}}=124 \mathrm{kPa}$.

Fig. 7 . - Typical direct shear paths at prescribed normal stiffness $(k)$; experience $\bullet$, simulation $\mathbf{\Lambda}$ dense Hostun sand, $\sigma_{n_{0}}=124 \mathrm{kPa}$.

Des simulations systématiques montrant l'effet de la densité initiale, de la contrainte normale initiale, et de la rigidité normale d'une part, du pas d'intégration d'autre part, ont été obtenues par MARCHINA [10] et comparées aux résultats expérimentaux d'HOTEIT [9]. La comparaison expérience simulation est d'autant moins satisfaisante que les conditions initiales sont proches des limites du domaine d'identification. La forme des réponses incrémentales à divers stades de l'intégration donne une idée de la qualité du modèle ; les résultats présentés (fig. 8) sont relativement « anguleux » car seuls quatre chemins élémentaires ont été utilisés (pas de chemins pseudo-œdométriques) et de plus les chemins à contrainte normale constante et à volume constant peuvent être peu éloignés.

\section{UNE APPLICATION PAR LA MÉTHODE DES ÉLÉMENTS FINIS}

La version précédente de ce modèle d'interface, utilisant deux chemins de base (en charge) a été utilisée dans un code éléments finis (méthode déplacements) en vue d'évaluer ses capacités de prédiction 

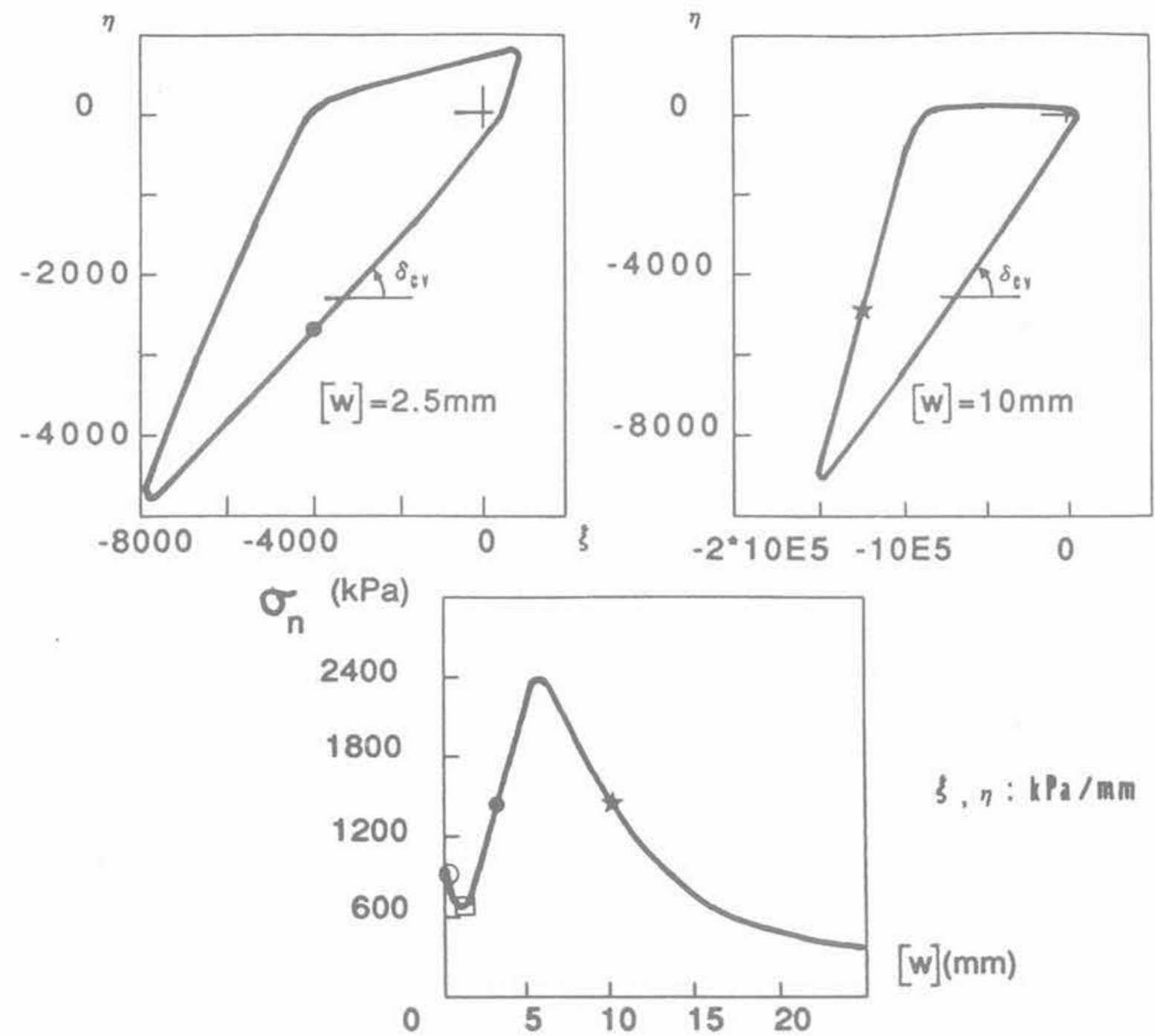

$[\mathrm{w}](\mathrm{mm})$
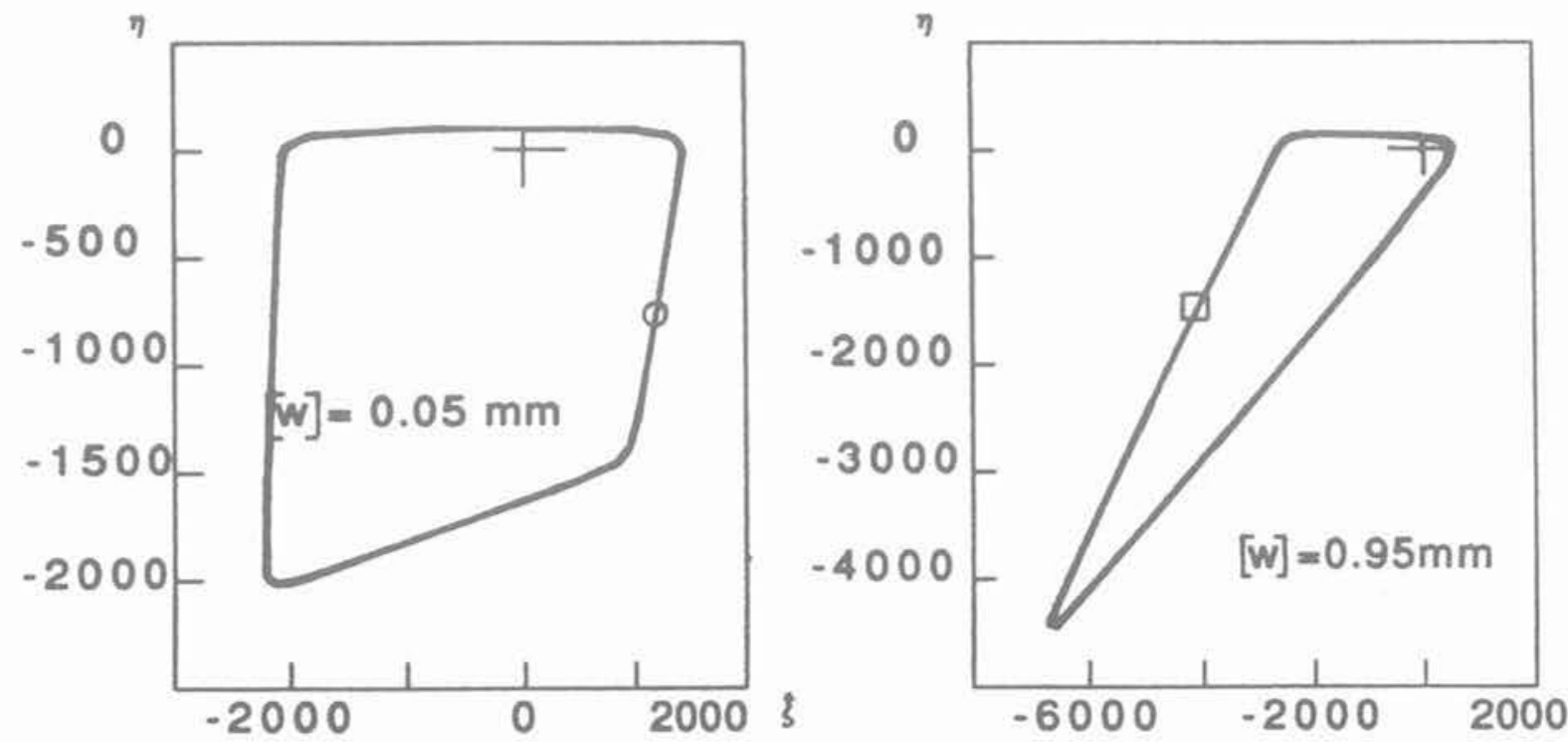

Fig. 8. - Quatre réponses incrémentales typiques (sable d'Hostun dense, $\sigma_{n_{0}}=1061 \mathrm{kPa}, k=5000 \mathrm{kPa} / \mathrm{mm}$ ). Fig. 8: - Four typicat incremental responses (dense Hostun sand, $\sigma_{n_{0}}=1061 \mathrm{kPa}, k=5000 \mathrm{kPa} / \mathrm{mm}$ ). 
(BOULON [1]). Nous avons modélisé le comportement d'un pieu modèle en tension ancré dans une cuve à sable. La figure 9 montre les conditions expérimentales et le maillage éléments finis utilisé pour la simulation numérique.

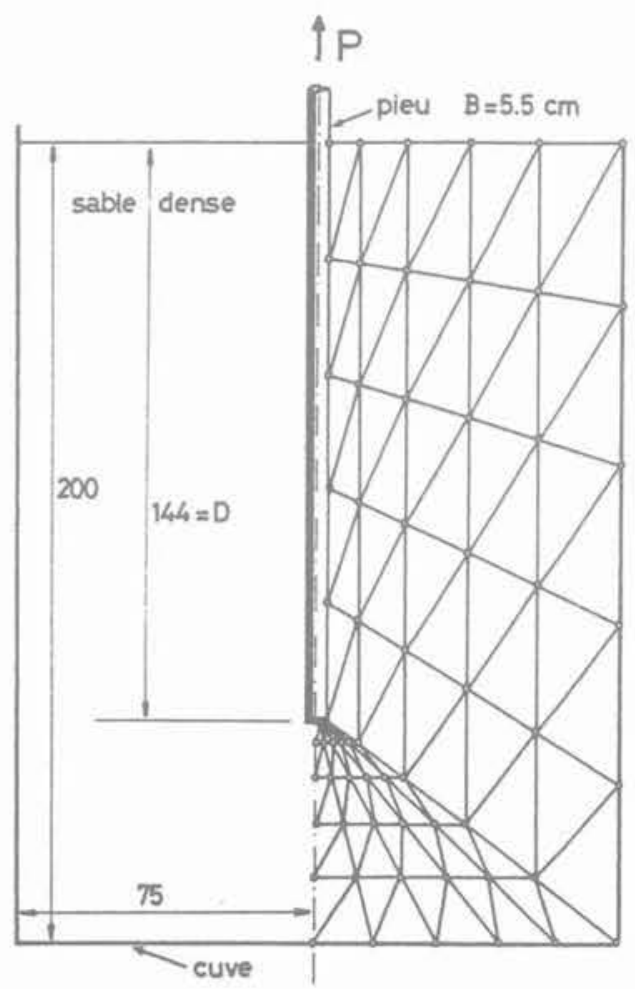

Fig. 9. - L'expérience de validation et le maillage éléments finis correspondant.

Fig. 9. - The experiment for validation and the corresponding FEM mesh.

Durant cette expérience, ont été mesurées, la force axiale à divers niveaux du pieu d'une part, et la contrainte normale agissant sur le fût à la profondeur $\mathrm{z}=1,44 \mathrm{~m}$ d'autre part; on passe aisément de l'effort normal au frottement latéral unitaire local par dérivation, ce qui permet d'accéder au coefficient de pression latérale des terres, K. Ce coefficient représente l'amplification de la contrainte normale agissant sur le fut, entre l'état Ko et l'état actuel. La figure 10 montre l'évolution de ce coefficient $\mathrm{K}$ en fonction du déplacement en tête du pieu, pour l'expérience (courbe (0)) et diverses simulations numériques (courbes (1) à (5)). L'expérience inclut des cycles dont l'enveloppe peut être considérée comme la courbe expérimentale à confronter aux simulations.

Le tableau 1 indique brièvement les types de comportements utilisés pour le sol et l'interface sol-pieu dans chaque simulation (le pieu est considéré comme élastique linéaire).

On remarquera qu'une valeur limite est obtenue pour $\mathrm{K}$ avec les calculs (2) à (4) - dont le calcul (4) avec la loi d'interface évoquée ci-dessus. Cet exemple montre qu'un modèle réaliste d'interface sol-structure nécessite la prise en compte d'une dilatance contractance non nulle, de niveau variant considérablement au cours d'un cisaillement donné. De plus, l'aspect

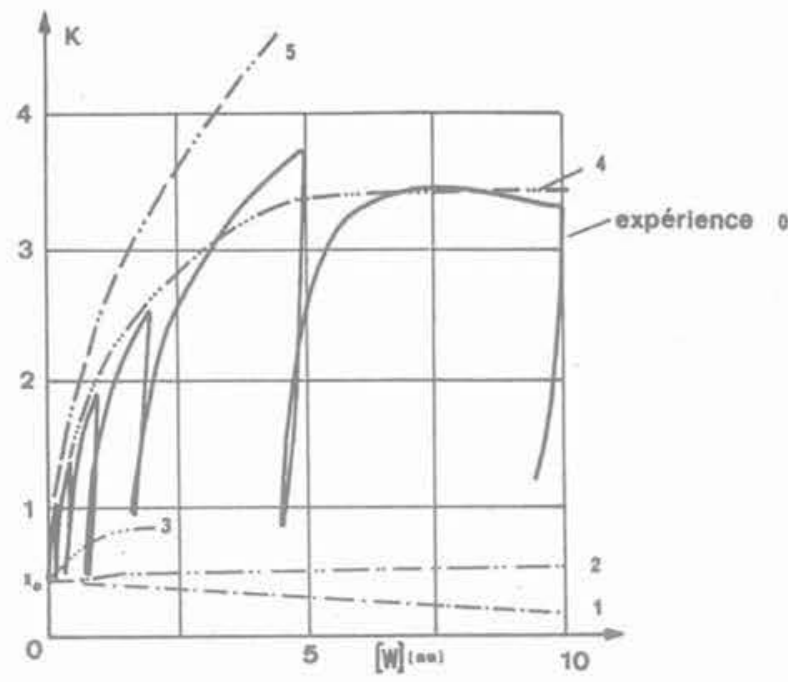

Fig. 10. - Extraction de pieu modèle ancré dans du sable grossier dense d'Hostun. Evolution du coefficient $K$ de pression latérale des terres (à la profondeur $z=1,44 \mathrm{~m}$ ). Expérience et simulations numériques.

Fig. 10 - Model pile in tension ensedided in a coarse dense Hostun sand.

Change in the $K$ coefficient of earth pressure Idepth $z=1.44$ m). Experiment and numerical simulations.

Tableau 1. - Lois de comportement utilisées pour le sol et pour l'interface sol-structure dans les simulations numériques (1) à (5).

Table 1. - Constitutive equations used for the soil and the soil-structure interface in the numerical simulation (1) to (5).

\begin{tabular}{|c|l|l|}
\hline $\begin{array}{c}\text { Simulation } \\
n^{\circ}\end{array}$ & \multicolumn{1}{|c|}{$\begin{array}{c}\text { Comportement } \\
\text { du sol }\end{array}$} & \multicolumn{1}{|c|}{$\begin{array}{c}\text { Comportement } \\
\text { d'interface }\end{array}$} \\
\hline 1 & élasticité linéaire & rigide \\
\hline 2 & élasticité linéaire & Coulomb, isovolume \\
\hline 3 & $\begin{array}{l}\text { directionnellement } \\
\text { dépendant }\end{array}$ & Coulomb, isovolume \\
\hline 4 & $\begin{array}{l}\text { élasticité } \\
\text { non linéaire }\end{array}$ & $\begin{array}{l}\text { directionnellement } \\
\text { dépendant }\end{array}$ \\
\hline 5 & $\begin{array}{l}\text { élastoplasticité } \\
\text { non standard }\end{array}$ & $\begin{array}{l}\text { élastoplasticité } \\
\text { non standard }\end{array}$ \\
\hline
\end{tabular}

le plus important du sol lui-meme affectant les variations du coefficient $\mathrm{K}$ est évidemment la compressibilité volumique, directement mobilisée sur "chemin pressiométrique $\$$; de ce dernier point de vue, de nombreux modèles de sols sont convenables.

\section{UN MODÈLE SIMPLIFIÉ DE PIEU SOUS CHARGEMENT AXIAL}

Les développements précédents permettent de proposer un modèle simplifié de comportement de pieu sous chargement axial. Soit une inclusion ancrée dans le sol orthotrope de révolution, l'axe de l'inclusion 
étant parallèle à l'axe privilégié d'orthotropie ; on suppose l'axisymétrie autour de cet axe et la déformation plane perpendiculairement à celui-ci, ainsi qu'une mise en place de l'inclusion par moulage in situ (sans expansion). Un cisaillement simple d'intensité rapidement décroissante avec la distance à l'axe se développe dans tout le plan radial au cours du chargement axial de l'inclusion. Supposons que le taux de changement de volume dû à ce cisaillement simple soit faible (c'est le cas pour un cisaillement simple selon les axes d'orthotropie) : le sol supporte alors principalement une sollicitation de type pressiométrique résultant du couplage entre les phénomènes normaux et tangentiels au sein de l'interface. Soit Ep le module pressiométrique (en petites déformations) du sol, supposé dans l'état Ko avant chargement (cf. fig. 11). Au terme d'un chargement axial, l'état de l'interface est représenté par les variables [w], [u], $\tau, \sigma_{\mathrm{h}}$.

L'écriture des compatibilités statique et cinématique à la frontière entre le sol et l'interface (rayon $\mathrm{R}+e$, $e \ll R)$ donne :

$$
E_{p}=\frac{\sigma_{R}-\sigma_{R_{0}}}{\frac{2 \pi R[u]}{\pi R^{2}}}
$$

En conséquence, le sol proprement dit agit sur le système interface-inclusion par sa rigidité latérale:

$$
\mathrm{k}=\frac{\sigma_{\mathrm{R}}-\sigma_{\mathrm{R}_{0}}}{[\mathrm{u}]} \simeq \frac{2 \cdot \mathrm{E}_{\mathrm{p}}}{\mathrm{R}}
$$

La grande importance des essais de cisaillement direct à rigidité normale imposée apparaît une fois de plus, car les courbes $(\tau,[w])$ issues de tels tests sont une bonne approximation des courbes $(\mathrm{t}-\mathrm{z})$ mondialement utilisée par les concepteurs de pieux. Des publications précédentes (BOULON [2]) ont réuni quelques données de base (expériences et simulations) sur ces courbes, en fonction de la contrainte normale initiale (profondeur) de la rigidité latérale $\mathrm{k}$ et de la densité initiale du matériau. HOTEIT [9] présente l'ensemble détaillé de ces résultats.

\section{CONCLUSION}

Le comportement d'interface sol-structure doit être pris en considération en tant que tel dans la simulation de l'interaction sol-structure, car la fine couche de contact entre sol et inclusion est sollicitée à un niveau de déformation sans commune mesure avec le reste du sol. La réponse de cet interface est clairement dépendante du chemin suivi et conduit à la mobilisation d'un « frottement apparent ». Les aspects cinématiques de ce comportement englobent toujours une phase dilatante durant une fraction du cisaillement, ce qui conduit à une modification des contraintes de contact. De plus, la rupture des grains a une incidence importante, conduisant fréquemment à la liquéfaction statique. La rigidité latérale imposée par le sol environnant à une inclusion, est un paramètre très significatif ; le frottement apparent résultant de ces deux facteurs (comportement d'interface sol-structure et rigidité latérale) peut être simulé physiquement en laboratoire grâce à des essais de cisaillement direct à rigidité normale imposée).

\section{REMERCIEMENTS}

Ces recherches ont été soutenues par le GRECO * Rhéologie des Géomatériaux * qui est vivement remercié.

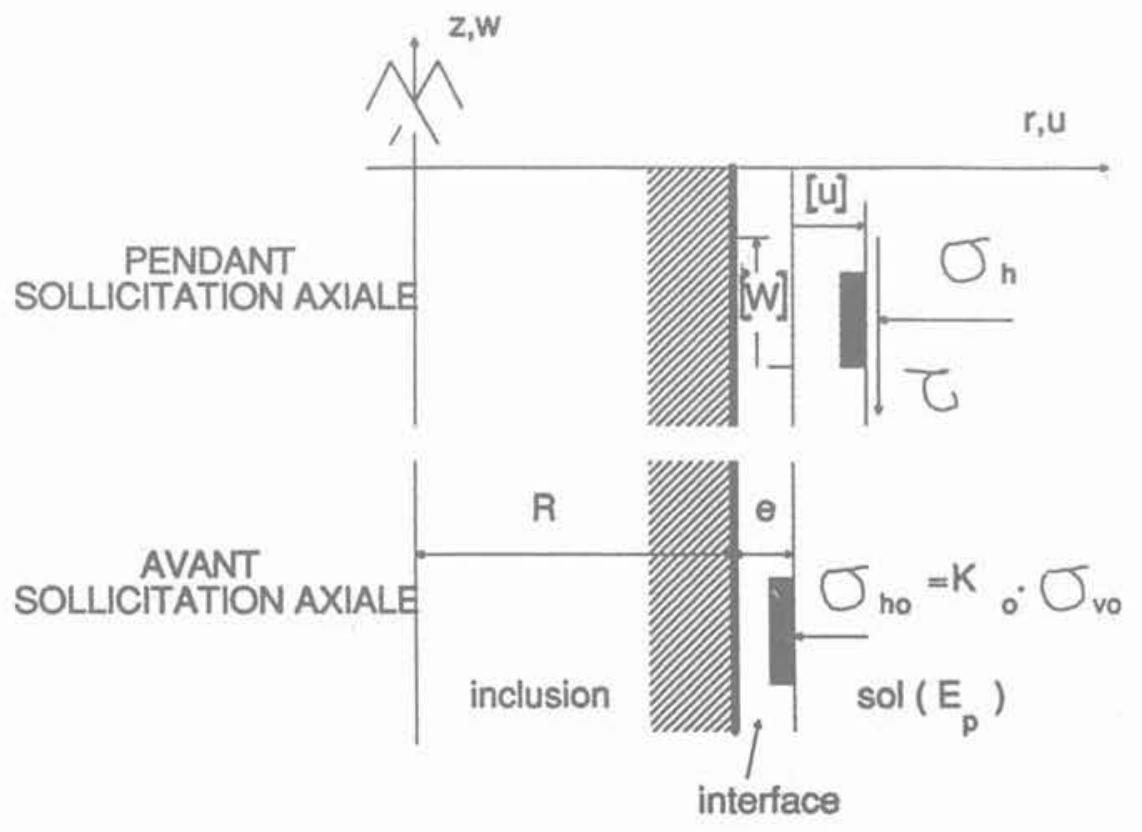

Fig. 11. - Modèle simplifié d'inclusion sous effort axial.

Fig. 11. - Simplified model for an inclusion under axial load. 


\section{BIBLIOGRAPHIE}

[1] BOULON M., HOTEIT N., MARCHINA P. (1988), A complete constitutive law for soilstructure interfaces. VI Int. conf. on num. methods in Geomechanics (ICONMIG), Innsbrück, Autriche, 1, 311-316.

[2] BOULON M. (1988), Numerical and physical modelling of piles behaviour under monotonous and cyclic loading. Int. Symposium SOWAS'88, Delft, Pays-Bas, 285-293.

[3] BOULON M. (1988), Contribution à la mécanique des interfaces sol-structure. Application au frottement latéral des pieux. Mémoire d'habilitation. Université de Grenoble.

[4] BRUMMUNDS W.F., LÉONARDS G.A. (1973), Experimental study of static and dynamic friction between sands and typical construction materials. J. testing and evaluation, 1, 2, 162-165.

[5] CLICHY W., BOULON M., DESRUES J. (1987), Etude expérimentale stéréophotogram. métrique des interfaces sols-fondations à la boite de cisaillement direct. $4^{\mathrm{e}}$ colloque franco-polonais de mécanique des sols appliqués, Grenoble, 311-325.

[6] COYLE H.M., CASTELLO R.R. (1981), New design correlations for piles in sand. J. Geotech. Eng. div. ASCE, 107, GT7, 965-985.

[7] FEDA J. (1976), Skin friction of piles. Proc. VI ECSMFE, Vienne, Autriche, 1.2, 423-428.

[8] GUILLOUX A., SCHLOSSER F., LONG N.T. (1979), Laboratory investigation of sand strip friction. Proc. int. conf. on soil reinforcement. Paris, $35-40$.

[9] HOTEIT N. (1990), Etude de cisaillement d'interface à rigidité normale imposée et appli. cations. Thèse, Université de Grenoble.
[10] MARCHINA P. (1989), Simulation numérique de l'interface sol-pieu par la méthode des équations intégrales aux frontières avec loi d'interface sol-structure. Thèse, Université de Grenoble.

[11] PLESHA M.E. (1987), Constitutive models for rock discontinuities with dilantancy and surface degradation. Int. Journ, for. num. and anal. meth. in Geomechanics, 11, 345-362.

[12] PUECH A., FORAY P., BOULON M., DESRUES J. (1979), Comportement et calcul des pieux à l'arrachement, application aux structures marines. Proc. 7th ECSMFE, Brighton, Grande-Bretagne, 1, 227-232.

[13] POTYONDY J.G. (1961), Skin friction between various soils and construction materials, Geotechnique, 2, 4, 339-353.

[14] SCARPELLI G., WOOD D.M. (1982), Experimental observations of shear band pattern in direct shear tests. IUTAM Symposium on deformation and failure of granular materials, Delft, 473-484

[15] SCHLOSSER F., GUILLOUX A. (1981), Le frottement dans le renforcement des sols. Revue française de Géotechnique, 16, 65-79.

[16] SCHLOSSER F., ELIAS V. (1978), Friction in reinforced earth, ASCE Convention, Pittsburgh, USA.

[17] WERNICK E. (1978), Skin friction of cylindrical anchors in non cohesive soils. Symp. on soil reinforcing and stabilizing techniques, Sydney, Australie, 201-219.

[18] YOSHIMI Y., KISHIDA T., A ring torsion apparatus for evaluation of friction between soil and metal surfaces. Geotechnical testing journal GTJODJ, 4, 4, 145-152. 